\title{
NEONATAL INHIBITION OF BRAIN ESTROGEN SYNTHESIS ALTERS ADULT NEURAL FOS RESPONSES TO MATING AND PHEROMONAL STIMULATION IN THE MALE RAT
}

\author{
J. BAKKER, ${ }^{*} \dagger$ M. J. BAUM $\ddagger$ and A. K. SLOB $\dagger$ \\ $\dagger$ Department of Endocrinology and Reproduction, Faculty of Medicine and Health Sciences, Erasmus \\ University, P.O. Box 1738, 3000 DR Rotterdam, The Netherlands \\ $\ddagger$ Department of Biology, Boston University, 5 Cummington Street, Boston, MA 02215, U.S.A
}

\begin{abstract}
Neonatal inhibition of brain estrogen formation in male rats by administration of the aromatase inhibitor, 1,4,6-androstatriene-3,17-dione (ATD), permanently changes aspects of their mating behavior and partner preference in adulthood. The medial preoptic area receives chemosensory inputs via a sexually dimorphic vomeronasal projection circuit, which responds to reproductively relevant pheromonal cues. The medial preoptic area also receives genital somatosensory inputs via the midbrain central tegmental field and the medial amygdala. We used Fos immunoreactivity as a marker of neuronal activation to determine whether there is a correspondence between the behavioral profiles of neonatally ATD-treated male rats and their neuronal responses in the medial preoptic area and other brain regions to somatosensory and chemosensory stimuli.

Achieving eight intromissions with an estrous female led to a greater neuronal Fos immunoreactivity in the medial preoptic area of neonatally ATD-treated male rats compared with neonatally cholesteroltreated male rats. Exposure for $1.5 \mathrm{~h}$ to chemosensory cues derived from soiled bedding of estrous females induced Fos immunoreactivity throughout the vomeronasal pathway (i.e. medial amygdala, bed nucleus of the stria terminalis and medial preoptic area) in both ATD and cholesterol males (Experiment 2a). By contrast, exposure for $1.5 \mathrm{~h}$ to chemosensory cues derived from soiled bedding of sexually active males revealed clear differences between ATD and cholesterol males in neuronal Fos immunoreactive (Experiment $2 \mathrm{~b}$ ). At peripheral portions of the vomeronasal pathway (i.e. the accessory olfactory bulb and the medial amygdala), there were no differences in the number of Fos-immunoreactivity neurons between ATD and cholesterol males. However, neurons in the more central portions of the vomeronasal pathway (i.e. the bed nucleus of the stria terminalis and the medial preoptic area) showed increased Fos immunoreactivity after exposure to odors from sexually active males in ATD males as opposed to cholesterol males. Females, like ATD males, showed neuronal Fos immunoreactivity at each level of the vomeronasal pathway after being exposed to odors from sexually active males.

These results suggest that the responsiveness of neurons in the central portion of the vomeronasal projection circuit to odors from sexually active males, but not estrous females, is sexually differentiated in male rats due to the neonatal action of estrogens. Copyright (C) 1996 IBRO. Published by Elsevier Science Ltd.
\end{abstract}

Key words: neonatal ATD treatment, Fos immunoreactivity, sexual behavior, pheromonal stimulation, preoptic area.

Perinatal administration of male rats of $1,4,6$ androstatriene-3,17-dione (ATD), which blocks the aromatization of testosterone to estradiol, affects the sexual differentiation of the CNS, as well as psycho-

*To whom correspondence should be addressed at: Department of Biology, Boston University, 5 Cummington Street, Boston, MA 02215, U.S.A. (present address).

Abbreviations: AOB, accessory olfactory bulb; ATD, 1,4,6androstatriene-3,17-dione; BNST, bed nucleus of the stria terminalis; CTF, central tegmental field; IR, immunoreactive, immunoreactivity; MePD, posterodorsal portion of the medial amygdala; MePV, posteroventral portion of the medial amygdala; $\mathrm{MPOA}$, medial preoptic area; PBS, phosphate-buffered saline; POA, preoptic area; SDN, sexually dimorphic nucleus. sexual differentiation. ${ }^{2,7,10,21,32,33}$ When tested in adulthood while gonadally intact, ATD-treated males show an altered sexual partner preference. When given free access to an estrous female and a sexually active male, they will approach, mount and intromit with the estrous female. However, they will also approach the active male, to whom they show proceptive behaviors (presenting, earwiggling, hopping and darting) and lordosis when mounted..$^{24,7}$ Control males, treated perinatally with cholesterol, prefer to mate with the estrous female and rarely approach the sexually active male. The most robust differences in partner preference between neonatally ATD-treated male and normal male rats were obtained when subjects were castrated as adults and subsequently treated with estradiol: ATD males showed a clear-cut 
preference for the sexually active male, whereas normal males preferred the estrous female. ${ }^{3}$

Numerous studies ${ }^{30}$ have shown that bilateral lesions of the medial preoptic area (mPOA) anterior hypothalamus continuum disrupt masculine coital function in male vertebrates and enhance males' proceptive and receptive responsiveness towards a stimulus male in cats, ${ }^{19}$ rats $^{20,24,26}$ and guinea-pigs, ${ }^{29}$ and induces a partner preference for a male in ferrets. ${ }^{27}$ Within the mPOA, a sexually dimorphic nucleus (SDN-POA), which is significantly larger in males than in females, was first described in rats. ${ }^{17}$ In rats, the sexual differentiation of the SDN-POA shows a close parallel with psychosexual differentiation, i.e. its larger size in males depends on the action of estrogenic metabolites of testosterone during a critical perinatal period. ${ }^{14,15,22}$ This correlation was emphasized by a recent study in which SDN-POA volume was reduced in both prenatally and pre- and neonatally ATD-treated males, with a larger reduction being achieved in the latter than in the former group. ${ }^{21}$

Immunocytochemical visualization of Fos, the nuclear protein product of the immediate early gene, $c$-fos, has provided useful information about the neural circuits which are activated following mating in male rats. ${ }^{5}$ In male rats, neural Fos immunoreactivity (IR) is increased after mating in several forebrain regions, including the $\mathrm{MPOA}$, bed nucleus of the stria terminalis (BNST) and posterodorsal portion of the medial amygdala (MePD), and in the midbrain central tegmental field (CTF). ${ }^{5,6,28,34} \mathrm{We}$ used Fos-IR as a marker of neural activity to reveal possible differences between neonatally estrogendeprived (i.e. neonatally ATD-treated) males and normal (i.e. neonatally cholesterol-treated) male rats' responses to genital and pheromonal stimulation associated with sexual behavior. In Experiment 1, the number of Fos-IR neurons was compared in several brain regions of adult male rats treated neonatally with ATD or cholesterol. Animals were killed $1 \mathrm{~h}$ after achieving eight intromissions with an estrous female.

In rodent species, reproductivity relevant pheromonal cues are detected by receptors in the vomeronasal organ, which in turn transmit this information centrally via the accessory olfactory bulb (AOB), the medial amygdala, BNST and $\mathrm{mPOA} .{ }^{23}$ In the rat, this vomeronasal projection pathway is sexually dimorphic, with males having more vomeronasal receptors and a larger number of neurons throughout. ${ }^{31}$ In male rats, the sexual differentiation of this vomeronasal pathway apparently occurs under the influence of perinatal estrogens. ${ }^{18,31}$ Therefore, in Experiment 2, we investigated whether pheromonal cues (derived from either the urine and feces of estrous females or sexually active males) may reveal differences in neural $c$-fos responses in the vomeronasal projection to the $\mathrm{mPOA}$ and/or the nucleus accumbens, a brain region which has been linked to reward and sexual motivation, ${ }^{16,25,35}$ between neonatally ATD-treated males and cholesterol-treated males.

\section{EXPERIMENTAL PROCEDURES}

Animals

Male and female Wistar RP rats bred in our laboratory were housed in single-sex groups of two to three. Food and water were available ad libitum. All rats were kept on a reversed $14: 10 \mathrm{~h}$ light-dark cycle (lights off from 7.45 a.m. to 5.45 p.m.). Female rats were time-mated and parturition occurred 22 days later. Within $2-4 \mathrm{~h}$ after birth, the newborn males received subcutaneously a silastic capsule (SR3: inner diameter $1.5 \mathrm{~mm}$, outer diameter $2.1 \mathrm{~mm}$, length $5 \mathrm{~mm}$ ) containing crystalline ATD or cholesterol under ice anesthesia. The implants were removed at 21 days of age (day of weaning), and the animals were housed two to three of the same treatment to a cage.

\section{Experiment 1: neural c-fos responses to mating}

At the age of approximately three months, 15 neonatally ATD-treated and 15 cholesterol-treated males were castrated under ether anesthesia and were injected daily with testosterone propionate ( $200 \mu \mathrm{g}$, s.c., in olive oil). The ATD and cholesterol males were pretested three times ( $30 \mathrm{~min} /$ test) with an estrous female in order to provide them with heterosexual experience. All behavioral testing was conducted in semicircular cages measuring $62 \times 40 \times 36 \mathrm{~cm}^{3}$ Before testing, the experimental animal spent a 15-min adaptation period in the cage. The test began when a female was put in the cage. The stimulus females were ovariectomized and brought into behavioral estrus by injecting $20 \mu \mathrm{g}$ of estradiol benzoate $24-48 \mathrm{~h}$ followed by $1.0 \mathrm{mg}$ of progesterone $3-4 \mathrm{~h}$ before testing.

All males were housed singly for one or two days prior to the actual experiment. Groups of ATD and cholesterol males were allowed to achieve eight intromissions with an estrous female. Then, these males were put singly in their home cages for $1 \mathrm{~h}$, after which they were perfused and their brains processed for Fos-IR. Pairs of ATD and cholesterol males were matched for number of mounts and intromissions, and testing time. Additional male rats (ATD and cholesterol; housed singly for one or two days) taken directly from their home cages served as unmated controls. In Experiment 1, 10 mated ATD, 10 mated cholesterol, five unmated ATD and five unmated cholesterol males were used.

Experiment $2 a$ : neural c-fos responses to chemosensory cues derived from soiled bedding of estrous females

At the age of approximately six months, five neonatally ATD-treated and 10 cholesterol-treated males were castrated under ether anesthesia using a midline abdominal incision and received estradiol subcutaneously through a silastic capsule (inner diameter $0.5 \mathrm{~mm}$, outer diameter $1.0 \mathrm{~mm}$, length $2.5 \mathrm{~cm}$ ). We chose adult estradiol treatment because the sexual behavior of ATD males differed most dramatically from that of control males under this hormonal condition. ${ }^{3}$ Two groups of ovariectomized females (six per group) were injected subcutaneously with estradiol benzoate $(20 \mu \mathrm{g}) 24-48 \mathrm{~h}$ prior to a progesterone $(1.0 \mathrm{mg})$ injection. Three hours later, all females were placed in clean cages which contained fresh sawdust. Bedding was collected $12 \mathrm{~h}$ later and immediately used in the experiment. The heterosexually experienced ATD and cholesterol males were housed singly for one to two days prior to use in the experiment in a room containing no other rats. Pairs of ATD and cholesterol males were perfused after spending $1.5 \mathrm{~h}$ in the cage containing soiled bedding from estrous females and their brains were processed for Fos-IR. Additional male rats, housed singly in a separate room 

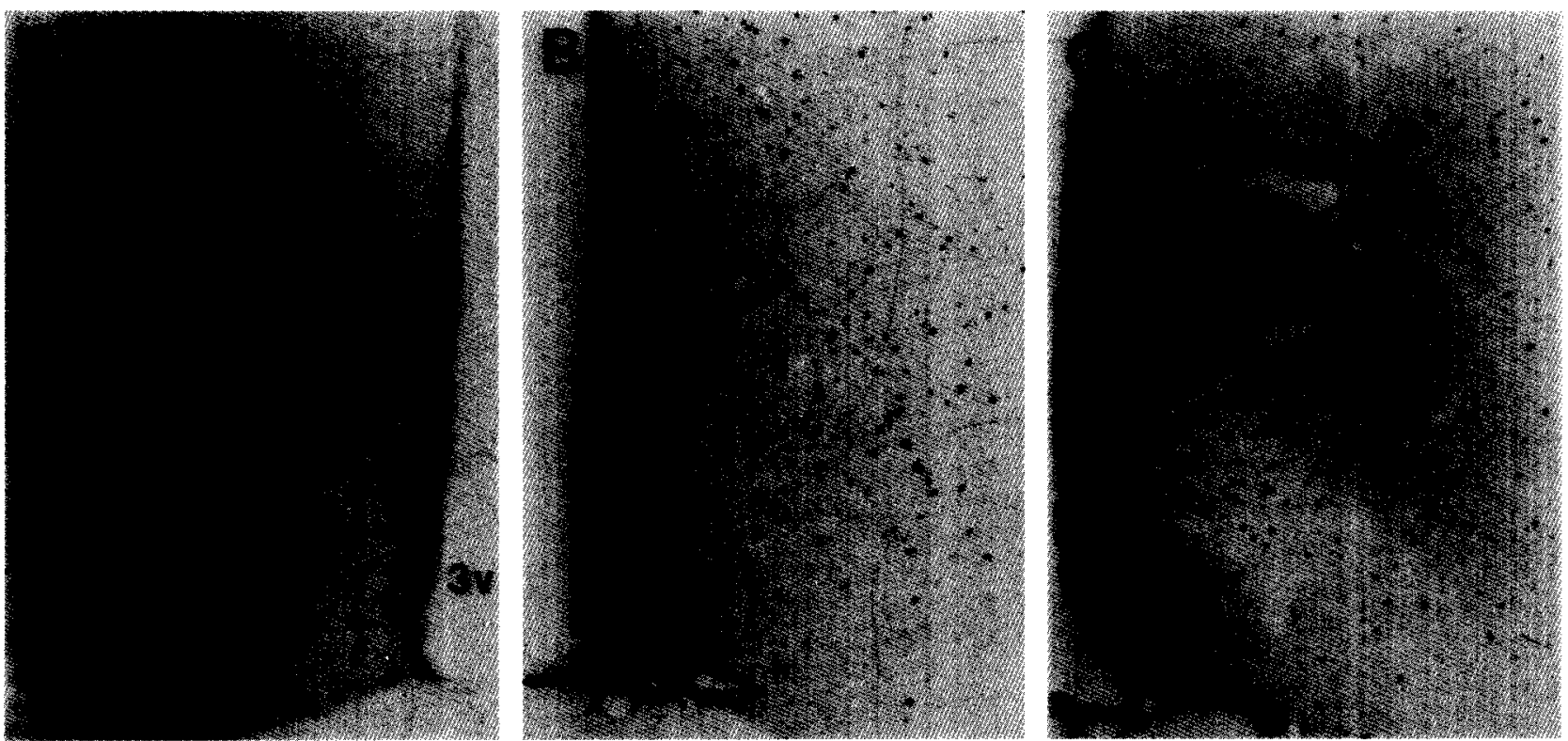

Fig. 1. Photomicrographs showing Fos-IR in the mPOA of an unmated cholesterol-treated male (A), a cholesterol-treated male after eight intromissions with an estrous female (B) and an ATD-treated male after eight intromissions with an estrous female (C). All males were castrated in adulthood and subsequently treated with testosterone propionate.

containing no other rats, were taken directly from their home cages to the perfusion room and served as controls not exposed to pheromones (home cage males). Because no differences in $c$-fos responses were found between the unmated ATD and cholesterol males in Experiment 1, we used only neonatally cholesterol-treated males as home cage males. All experiments took place in the early part of the dark phase of the light-dark cycle (between 8.30 and 11.30 a.m.).

Experiment $2 b$ : neural c-fos responses to chemosensory cues derived from soiled bedding of sexually active males

At the age of approximately nine months, seven neonatally ATD-treated and seven cholesterol-treated males were castrated under ether anesthesia using a midline abdominal incision and received estradiol subcutaneously through a silastic capsule (inner diameter $0.5 \mathrm{~mm}$, outer diameter $1.0 \mathrm{~mm}$, length $2.5 \mathrm{~cm}$ ). The female rats used in this experiment were ovariectomized and treated with estradiol benzoate $24-48 \mathrm{~h}$ prior to the experiment. The ATD and cholesterol males were pretested twice with an estrous female $(15 \mathrm{~min} / \mathrm{test})$ and twice with an active male (15 min/test) in order to provide them with sexual experience. The females had been used as stimulus animals during partner preference testing earlier in our laboratory.

Three groups of males (six per group) were placed in clean cages which contained fresh sawdust. Bedding was collected $72 \mathrm{~h}$ later and immediately used in the experiment. All experimental animals (ATD, cholesterol and females) were housed singly one or two days prior to use in the experiment in a room containing no other rats (females separate from males). The experimental animals were perfused after spending $1.5 \mathrm{~h}$ in the cage containing soiled bedding from active males and their brains were processed for Fos-IR. A control group, comprising two ATD males, two cholesterol males and two females, was killed after spending $1.5 \mathrm{~h}$ in clean bedding (clean bedding subjects). We chose this particular composition of the control group because: (i) no differences in Fos-IR were found between unmated ATD and cholesterol males in Experiment 1 ; (ii) neural $c$-fos responses in

Table 1. Effect of eight intromissions with an estrous female on the mean ( \pm S.E.) number of Fos-immunoreactive neurons (counted per standard area, $0.27 \mathrm{~mm}^{2}$ ) in neonatally estrogen-deprived (ATD-treated) and normal males (cholesterol-treated)

\begin{tabular}{|c|c|c|c|c|c|c|c|c|}
\hline \multirow[b]{2}{*}{ Group } & \multirow[b]{2}{*}{$n$} & \multicolumn{2}{|c|}{ Nucleus accumbens } & \multirow[b]{2}{*}{ mPOA } & \multirow[b]{2}{*}{ SDN-POA $\ddagger$} & \multirow[b]{2}{*}{ BNST } & \multirow[b]{2}{*}{ MePD } & \multirow[b]{2}{*}{ CTF } \\
\hline & & Core & Shell & & & & & \\
\hline \multicolumn{9}{|c|}{$\begin{array}{l}\text { Experiment 1: eight intromissions with female } \\
\text { Unmated }\end{array}$} \\
\hline $\begin{array}{l}\text { Cholesterol } \\
\text { ATD }\end{array}$ & $\begin{array}{l}5 \\
5\end{array}$ & $\begin{array}{r}9 \pm 3 \\
10 \pm 3\end{array}$ & $\begin{array}{l}5 \pm 1 \\
6 \pm 1\end{array}$ & $\begin{array}{r}8 \pm 2 \\
20 \pm 4\end{array}$ & $\begin{array}{l}2 \pm 1 \\
4 \pm 2\end{array}$ & $\begin{array}{r}8 \pm 3 \\
10 \pm 2\end{array}$ & $\begin{array}{l}19 \pm 6 \\
16 \pm 5\end{array}$ & $\begin{array}{l}6 \pm 2 \\
2 \pm 1\end{array}$ \\
\hline $\begin{array}{l}\text { Mated } \\
\text { Cholesterol } \\
\text { ATD }\end{array}$ & $\begin{array}{l}10 \\
10\end{array}$ & $\begin{array}{l}62 \pm 11^{*} \\
71 \pm 13^{*}\end{array}$ & $\begin{array}{l}52 \pm 9^{*} \\
64 \pm 8^{*}\end{array}$ & $\begin{array}{l}119 \pm 9^{*} \\
181 \pm 17^{*} \dagger\end{array}$ & $\begin{array}{l}23 \pm 5^{*} \\
43 \pm 5^{* \dagger}\end{array}$ & $\begin{array}{l}66 \pm 8^{*} \\
84 \pm 8^{*}\end{array}$ & $\begin{array}{r}92 \pm 9^{*} \\
104 \pm 7^{*}\end{array}$ & $\begin{array}{l}25 \pm 5^{*} \\
34 \pm 5^{*}\end{array}$ \\
\hline
\end{tabular}

All animals were castrated and treated with testosterone propionate at the time of the experiment.

*Significantly $(P<0.05)$ higher compared to unmated males

†Significantly $(P<0.05)$ higher in mated ATD males compared to mated cholesterol-treated males.

†SDN-POA data are expressed as mean number of Fos-IR neurons inside the SDN-POA per mean SDN-POA area. 


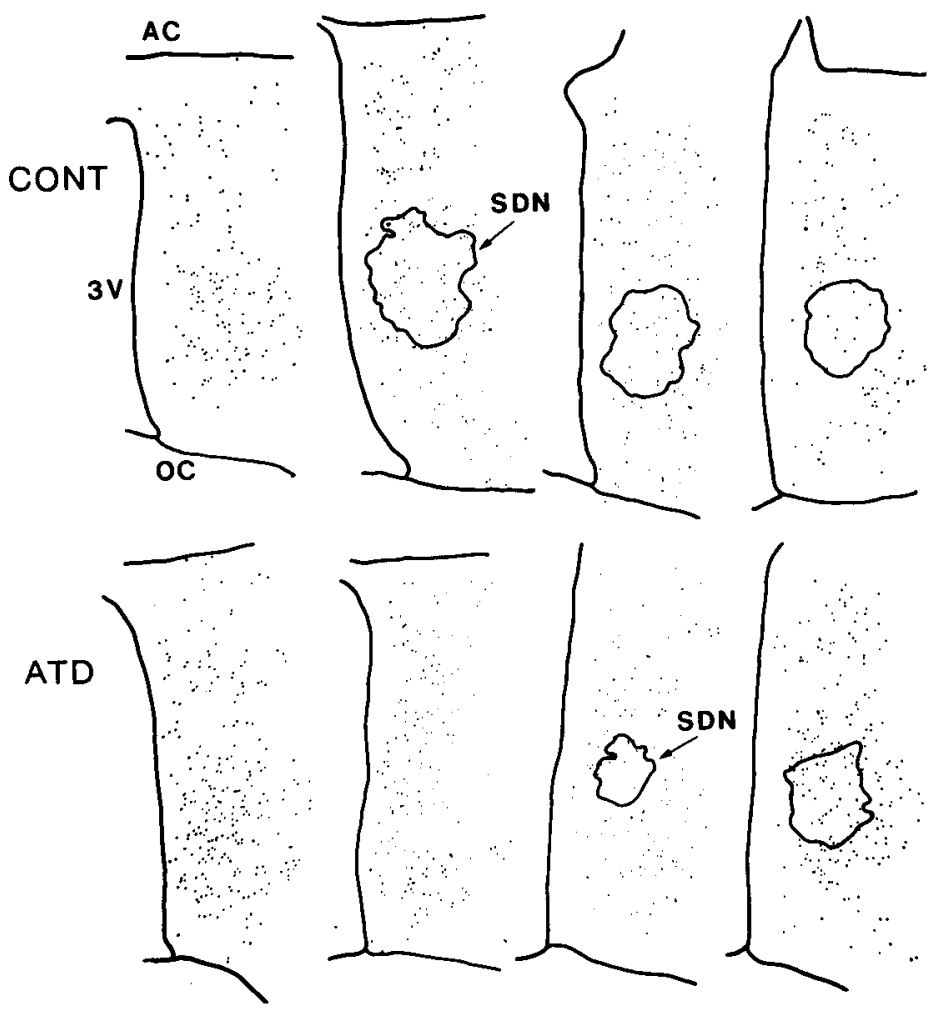

Fig. 2. Camera lucida drawings of Fos-IR nuclei (black dots) and the SDN in consecutive coronal sections through the POA of a representative cholesterol-treated male (CONT) and an ATD-treated male after eight intromissions with an estrous female.

home cage subjects in Experiment 2a were very low; (iii) females have been added to the experimental groups. All experiments took place in the early part of the dark phase of the light-dark cycle (between 8.00 and 11.00 a.m.).

\section{Immunocytochemistry}

Rats were anesthetized with sodium pentobarbital $(100 \mathrm{mg} / \mathrm{rat}$, i.p.), given an intracardiac injection of heparin (1000 U/rat) and perfused via the aorta with $0.1 \mathrm{M}$ phosphate-buffered saline (PBS; pH 7.3) followed by 4\% paraformaldehyde in $0.1 \mathrm{M}$ phosphate buffer ( $\mathrm{pH} 7.3$ ). Brains were removed and postfixed in $4 \%$ paraformaldehyde for $2 \mathrm{~h}$ before being placed in $20-30 \%$ sucrose-PBS for cryoprotection overnight on a shaker at $4^{\circ} \mathrm{C}$. Coronal sections of $52 \mu \mathrm{m}$ were cut using a sledge microtome with a freezing stage. In Experiments 1 and $2 a$, consecutive sections were saved at each of four levels, including the nucleus accumbens, mPOA/BNST, medial amygdala and midbrain CTF (in Experiment 1 only). In Experiment $2 b$, consecutive sections were saved at each of three levels, including the olfactory bulb, the nucleus accumbens and from the medial preoptic anterior hypothalamus to the MePD. Free-floating sections were rinsed twice in $0.1 \mathrm{M}$ PBS and then incubated overnight on a shaker at room temperature with an anti-Fos antiserum raised in rabbit against the $\mathrm{N}$-terminal sequence of rat Fos amino acids (DCH-1; a gift from Dr David Hancock, London, U.K.). After incubation in primary antiserum, the brain sections were rinsed four times in $0.1 \mathrm{M}$ PBS containing $0.02 \%$ Triton X-100 and incubated for $2 \mathrm{~h}$ on a shaker at room temperature with biotinylated goat anti-rabbit immunoglobulin G. After incubation with the secondary antiserum, the sections were rinsed four times and incubated for $1.5 \mathrm{~h}$ on a shaker at room temperature with avidin-biotin-peroxidase complex solution (ABC Elite Kit,
Vector Laboratories). The brain sections were again rinsed four times in 0.1 M PBS (no Triton X-100) and then reacted with nickel chloride-3,3'-diaminobenzidine and $0.0003 \%$ hydrogen peroxide for 5-10 min (DAB Kit, Vector Laboratories). The sections were rinsed three times, mounted onto gelatin-coated slides and coverslipped using Permount. In Experiment 1, after the immunocytochemistry for Fos protein was completed, alternate sections were counterstained with Cresyl Violet in order to determine the boundaries of the SDN.

\section{Analysis}

To quantify the numbers of Fos-IR nuclei, all slides were coded so that the investigator had no knowledge of sex or treatment condition of brain sections from any subject. In Experiment 1, two brain sections at the level of the nucleus accumbens, mPOA, BNST, MePD and CTF were selected for quantitative analysis for each animal. In Experiment 2a, brain sections at the level of the nucleus accumbens, $\mathrm{mPOA}$, BNST, posteroventral portion of the medial amygdala (MePV) and MePD were selected for quantitative analysis for each animal. We did not collect the olfactory bulbs in Experiment 2a, because Bressler and Baum ${ }^{8}$ reported similar increments in the number of Fos-IR neurons in the olfactory bulb for males and females. Thus, we had no expectation of finding a difference in Experiment $2 \mathrm{a}$. In Experiment $2 \mathrm{~b}$, brain sections at the level of the AOB (mitral and granule cell layer), nucleus accumbens, mPOA, BNST, MePV and MePD were selected for quantitative analysis for each animal. All of the Fos-IR nuclei in a field of view under the $\times 25$ objective $\left(0.27 \mathrm{~mm}^{2}\right)$ were drawn using a camera lucida and counted later.

To analyse the distribution of Fos-IR nuclei in the SDN-POA (Experiment 1), the number of Fos-IR nuclei inside the SDN was counted for each animal in the 
counter-stained sections. Because the SDN was present in one to three sections only, the mean number of Fos-IR nuclei inside the SDN was calculated for each animal. Area measurements of the SDN (coronal sections) were performed unilaterally using the method described by Houtsmuller et $a l^{21}$ and a mean SDN area was calculated for each animal. The Fos-IR data of the SDN are expressed as mean number of Fos-IR nuclei inside the SDN/mean SDN area. In some animals (two cholesterol unmated, one ATD and one cholesterol mated), the sections taken for the mPOA/BNST did not include the SDN. These animals were excluded from statistical analysis. In addition, camera lucida drawings of Fos-IR nuclei and the SDN were made of consecutive coronal sections through the MPOA of a representative ATD male and a cholesterol male after mating.

\section{Statistics}

All Fos-IR data were first analysed for normal distribution using the Kolmogorov-Smirnov test and were subsequently subjected to two-way ANOVA in Experiment 1 (neonatal treatment $\times$ mating) or one-way ANOVA in Experiment 2 (groups), followed by the StudentNewman-Keuls method for pairwise multiple comparison procedures (Student-Newman-Keuls method). If the normality test failed $(P<0.05)$, the data were subjected to Kruskal-Wallis one-way ANOVA on ranks, followed by the Student-Newman-Keuls method for pairwise multiple comparison procedures. In Experiment 1, the sections containing the nucleus accumbens were taken too caudal for some animals (two ATD and one cholesterol unmated, one ATD and one cholesterol mated). These animals were excluded from statistical analysis.

\section{RESULTS}

\section{Experiment 1: neural c-fos responses to mating}

Mounting and intromitting with an estrous female significantly augmented the number of Fos-IR neurons in several brain regions of both ATD and cholesterol males. The magnitude of this $c$-fos response was very similar in ATD and cholesterol males, with the exception of the response in the MPOA, which was greater in ATD males than in cholesterol males. An example of the effect of mating on Fos-IR in the mPOA is shown in Fig. 1. Two-way ANOVA showed that there was a significant effect of mating $[F(1 / 26)=80.9, P<0.0001]$ and a significant effect of neonatal treatment $[F(1 / 26)=5.9, P=0.02]$ on the number of Fos-IR nuclei in the mPOA (Table 1). Post hoc analyses revealed that there were significantly more Fos-IR nuclei in the mPOA of mated males than of unmated males. Furthermore, mated ATD males had significantly more Fos-IR nuclei in the mPOA than mated cholesterol males. Mating also augmented the number of Fos-IR nuclei in the BNST $[F(1 / 26)=57.2, P<0.0001]$, MePD $[F(1 / 26)=72.8$, $P<0.0001]$ and CTF $[F(1 / 26)=21.9, P<0.0001]$ (Table 1) to equivalent degrees in ATD and cholesterol males. Finally, the nucleus accumbens shell $[F(1 / 20)=28.2, P<0.0001]$ and core $[F(1 / 20)=15.0$, $P=0.001]$ also showed increased Fos-IR after mating in both ATD and cholesterol males (Table 1).

The mating-induced increments in Fos-IR occurred in portions of the MPOA which included, but were not restricted to, the SDN. Camera lucida

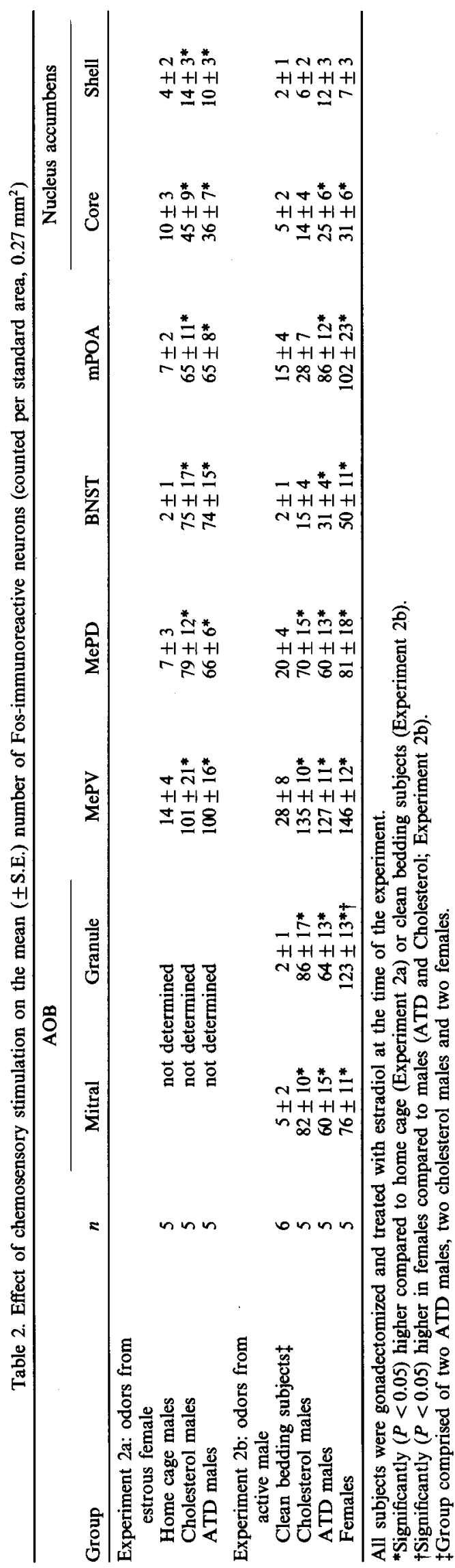


drawings of Fos-IR neurons and the SDN in consecutive coronal sections through the $\mathrm{mPOA}$ of mated males are shown in Fig. 2. Two-way ANOVA (neonatal treatment $\times$ mating) on the mean number of Fos-IR neurons inside the SDN (see Table 1) showed a significant effect of mating $[F(1 / 22)=29.4$, $P<0.0001]$ and of neonatal treatment $[F(1 / 22)=4.2$, $P=0.05]$ on the number of Fos-IR nuclei in the SDN. Post hoc analyses revealed that the number of Fos-IR nuclei was significantly higher in mated males than in unmated males. Furthermore, the SDN of mated ATD males contained more Fos-IR neurons than the SDN of mated cholesterol males. There were no significant differences between ATD and cholesterol males in mean SDN area (ATD: $0.066 \pm 0.005 \mathrm{~mm}^{2}$; cholesterol: $0.078 \pm 0.007 \mathrm{~mm}^{2}$ ).

Experiment $2 a$ : neural c-fos responses to chemosensory cues derived from soiled bedding of estrous females

Exposure to soiled bedding of estrous females significantly augmented the number of Fos-IR nuclei at each level of the vomeronasal projection circuit of both ATD and cholesterol males, compared to home cage control males. The magnitude of this $c$-fos response was very similar in ATD and choles- terol males. One-way ANOVA revealed that exposure to pheromonal cues of estrous females augmented the number of Fos-IR neurons in the $\mathrm{MePV}[F(2 / 12)=8.1, \quad P=0.006]$ and MePD $[F(2 / 12)=18.6, P=0.0002]$ to equivalent numbers in ATD and cholesterol males (Table 2). Pheromonal stimulation also increased Fos-IR in the BNST $[F(2 / 12)=8.1, P=0.006]$ and $\operatorname{mPOA}[F(2 / 12)=$ 15.1, $P=0.0005]$ similarly in ATD and cholesterol males (Table 2). Furthermore, there was a significant pheromonal-induced increment in Fos-IR in the nucleus accumbens core $[F(2 / 12)=5.9, P=0.02]$ and shell $[F(2 / 12)=4.2, P=0.04]$ to equivalent degrees in ATD and cholesterol males (Table 2).

Experiment $2 b$ : neural c-fos responses to chemosensory cues derived from soiled bedding of sexually active males

There were clear differences between ATD and cholesterol males in neuronal $c$-fos expression following exposure to soiled bedding of sexually active males. At peripheral stages of the vomeronasal pathway (i.e. the AOB and MePD), ATD and cholesterol males showed similar $c$-fos responses, but at more central levels of the vomeronasal pathway (i.e. the
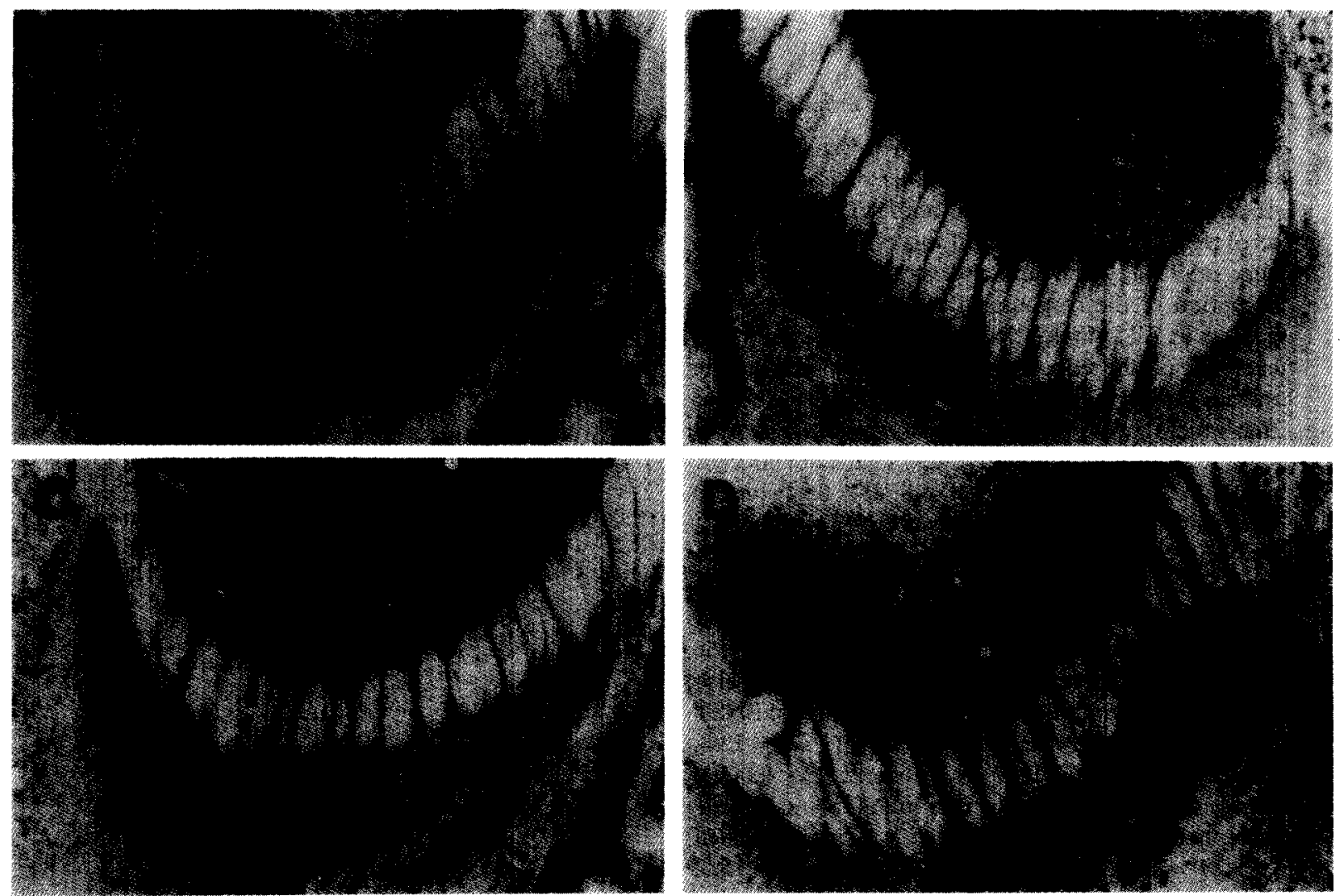

Fig. 3. Photomicrographs of the AOB showing FOS-IR neurons in the mitral (m) and granular (gr) layers of a male after spending $1.5 \mathrm{~h}$ on clean bedding (A), a cholesterol-treated male after spending $1.5 \mathrm{~h}$ on soiled bedding from sexually active males (B), an ATD-treated male after spending $1.5 \mathrm{~h}$ on soiled bedding from sexually active males (C) and a female after spending $1.5 \mathrm{~h}$ on soiled bedding from sexually active males (D). All animals were gonadectomized in adulthood and subsequently treated with estradiol. 
BNST and mPOA), ATD males still showed clear $c$-fos responses, whereas cholesterol males did not differ from subjects exposed only to clean bedding. Female rats, like ATD males, showed $c$-fos responses at each level of the vomeronasal pathway after being exposed to soiled bedding of sexually active males. Examples of the effect of pheromonal stimulation on Fos-IR in the mitral and granule cell layer of the AOB (peripheral level of the vomeronasal pathway) and in the mPOA (central level of the vomeronasal pathway) are shown in Figs 3 and 4. One-way
ANOVA showed that exposure to soiled bedding from active males significantly augmented the number of Fos-IR nuclei in the mitral cell layer of the AOB $[F(3 / 13)=10.1, P=0.001]$ to equivalent numbers in males (ATD and cholesterol) and females (Table 2). The number of Fos-IR was also augmented in the granule cell layer of the $\mathrm{AOB}[F(3 / 13)=15.1$, $P<0.001]$ in males (ATD and cholesterol) and females, with females showing a higher $c$-fos response than ATD and cholesterol males. Pheromonal stimulation also augmented Fos-IR in the MePV
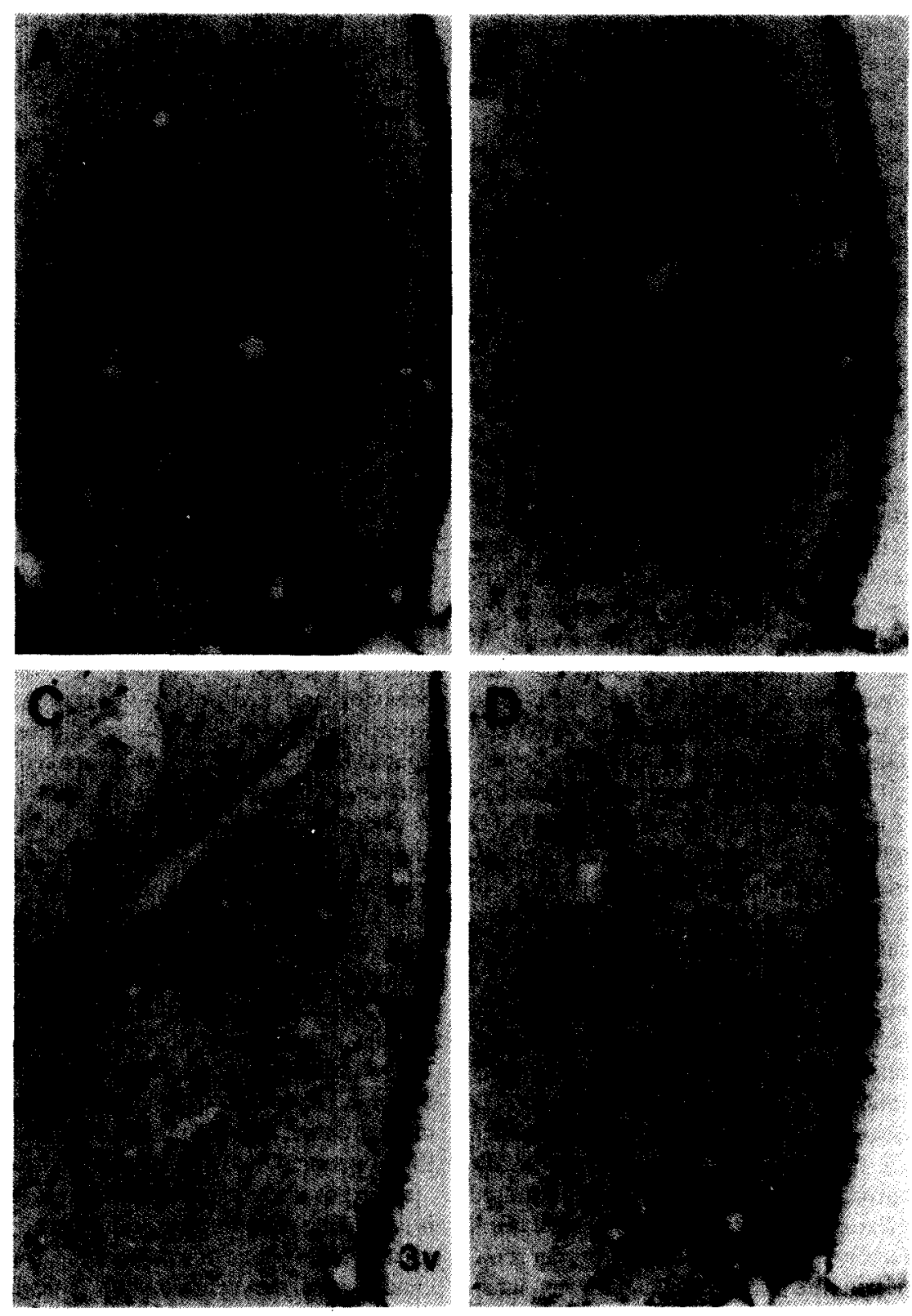

Fig. 4. Photomicrographs of Fos-IR in the mPOA of a male after spending $1.5 \mathrm{~h}$ on clean bedding (A), a cholesterol-treated male after spending $1.5 \mathrm{~h}$ on soiled bedding from sexually active males (B), an ATD-treated male after spending $1.5 \mathrm{~h}$ on soiled bedding from sexually active males (C) and a female after spending $1.5 \mathrm{~h}$ on soiled bedding from sexually active males (D). All animals were gonadectomized in adulthood and subsequently treated with estradiol. 
$[F(3 / 17)=26.0, \quad P<0.0001]$ and the MePD [Kruskal-Wallis one-way ANOVA on ranks: $H=8.5$ (d.f. $=3$ ), $P=0.04$ ] to similar numbers in both sexes. At more central levels in the vomeronasal pathway, however, there were clear differences between ATD and cholesterol males in $c$-fos responses to odors from active males in the BNST [Kruskal-Wallis one-way ANOVA on ranks: $H=14.9$ (d.f. $=3$ ), $P=0.002]$ and $\mathrm{mPOA}[F(3 / 17)=8.8, P=0.001]$, with ATD males showing significantly larger numbers of Fos-IR nuclei in the BNST and mPOA compared to cholesterol males and clean bedding subjects. Cholesterol males showed no responsiveness in the $\mathrm{mPOA}$ and BNST, equivalent to clean bedding subjects. Females showed similar amounts of Fos-IR in the BNST and mPOA as ATD males (Table 2). Exposure to soiled bedding of sexually active males also augmented the number of Fos-IR in the nucleus accumbens core in both females and ATD males $[F(3 / 16)=5.9, P=0.006]$. Cholesterol males did not show a significant increase in Fos-IR in the nucleus accumbens core.

\section{DISCUSSION}

\section{Neural c-fos responses to pheromonal stimulation}

The most interesting finding of the present study was the sexually dimorphic $c$-fos response in neurons of the vomeronasal pathway to chemosensory cues derived from the soiled bedding of sexually active males. Neonatal inhibition of brain estrogen synthesis in males made their $c$-fos responses in the vomeronasal pathway more similar to females than to control males. In peripheral portions of the vomeronasal pathway (i.e. the $A O B, M e P D$ and MePV), there were no differences between ATD and cholesterol males in the number of Fos-IR neurons. In more central portions of the vomeronasal pathway (i.e. the BNST and $\mathrm{mPOA}$ ), differences between ATD and cholesterol males became evident. In ATD males, the BNST and $\mathrm{mPOA}$ were clearly responsive to odors derived from sexually active males, whereas in cholesterol males these brain areas were not responsive. The peripheral $c$-fos response in the cholesterol males indicates that at least they detected the malederived odors. However, the lack of a central $c$-fos response indicates that the signal was differentially processed in cholesterol males as opposed to ATD males. ATD males resembled female subjects, which also showed $c$-fos responses at each level of the vomeronasal projection pathway after being exposed to odors of sexually active males. The behavioral findings of earlier studies ${ }^{3,11,12}$ predict the neuronal $c$-fos responses in the vomeronasal pathway when exposed to odors from sexually active males. When tested for sexual partner preference (choice: estrous female vs sexually active male), both female ${ }^{11,12}$ and neonatally ATD-treated males, ${ }^{3}$ gonadectomized and treated with estradiol in adulthood, spent signifi- cantly more time approaching and interacting sexually with a sexually active male than with an estrous female. By contrast, cholesterol-treated control males, ${ }^{3}$ castrated and estradiol-treated in adulthood, spent significantly more time approaching and interacting sexually with an estrous female than with a sexually active male. Thus, there is a sexual dimorphism in partner preference which is dependent on the neonatal action of estrogen in males. The sexual dimorphism in partner preference corresponds with the sexual dimorphism in the functional activity of the vomeronasal projection pathway, as revealed by Fos-IR, following exposure to odors from sexually active males. The sexual differentiation of the vomeronasal responses to odors of sexually active males is mediated by the neonatal action of estrogen in male subjects.

By contrast, the distribution and intensity of the increments in neuronal Fos-IR following exposure to chemosensory cues derived from the soiled bedding of estrous females was very similar in ATD and cholesterol males. Chemosensory cues derived from estrous females' bedding augmented Fos-IR in peripheral as well as in central portions of the vomeronasal projection circuit. Thus, there was no effect of neonatal estrogen deprivation on male rats' later neuronal responsiveness to odors from estrous females. From our previous behavioral findings, ${ }^{3}$ we expected that Fos-IR would be lowered in ATD males, because ATD males seem to be less motivated than cholesterol males to seek out the estrous female. Apparently, the behavioral findings of this previous study do not predict the neuronal $c$-fos responses in the chemosensory pathway when exposed to odors from estrous females. A similar discrepancy between behavior and neuronal $c$-fos responses was found recently by Bressler and Baum. ${ }^{8}$ These authors investigated odor preference (urine-soaked bedding from estrous vs anestrous females) and neuronal $c$-fos responses to soiled bedding from estrous and anestrous females in gonadectomized, testosterone propionate-treated male and female rats. Both males and females preferred to investigate the estrous bedding for more time than the anestrous bedding, although they had similar increments in Fos-IR after exposure to bedding from either estrous or anestrous females at each level of the vomeronasal pathway. ${ }^{8}$ This result was not predicted by the behavioral findings. Although partner preference is sexually differentiated, there is no clear sexual dimorphism in the functional activity of the vomeronasal system after exposure to odors from estrous females, which is surprising since large morphological sex differences exist at all levels of the pathway. ${ }^{31}$ The sexual differentiation of the vomeronasal pathway in males is influenced by the presence of perinatal androgens. ${ }^{31}$ Neonatal castration of male rats decreases the num. ber of cells and/or volumetric measures in the vomeronasal organ, AOB, the medial posterior division of the BNST and the bed nucleus of the 
olfactory tract. Neonatal androgenization of females makes their vomeronasal pathway more male-like. ${ }^{31}$ These effects of testosterone on the developing vomeronasal projection circuit are probably mediated by its estrogenic metabolite, as has already been shown for the medial amygdala, AOB and bed nucleus of the olfactory tract. ${ }^{31}$ As shown in the present study, the vomeronasal system of ATD males functionally resembles that of females. The morphology of this system has not yet been studied in ATD males, however, except for the mPOA. In future studies, it will be important to determine whether neonatal ATD treatment blocks the masculinization of additional portions of the vomeronasal pathway (i.e. the AOB, medial amygdala and BNST) in male rats.

\section{Neural c-fos responses to mating}

Mounting and intromitting with an estrous female led to a greater neuronal $c$-fos expression in the mPOA of male rats, in which brain estrogen synthesis was inhibited neonatally by ATD treatment. Thus, neurons in the MPOA of ATD males, in which the normal processes of estrogen-dependent masculinization and defeminization of sexual behavior were attenuated, seem to be more responsive to mounting and intromissive stimulation than those in the MPOA of cholesterol males. The significance of the higher $c$-fos expression in the mPOA of neonatally ATDtreated male rats is not obvious. One possibility is that the display of masculine sexual behavior activated both stimulatory and inhibitory neurons involved in the regulation of sexual behavior in the mPOA of ATD males, whereas in cholesterol males only stimulatory neurons were activated. Supportive evidence includes the observation that ATD males first mount and intromit with an estrous female as quickly as cholesterol males. However, as the pair test progresses, the rate of mounting and intromitting decreases in ATD males, but not in cholesterol males. $^{2}$ The cholesterol males continue to copulate until ejaculation is achieved. This decreased rate of mounting and intromitting in ATD males might explain why ATD males do not ejaculate as readily as cholesterol males. ${ }^{2-4,7}$

After eight intromissions with an estrous female, the number of Fos-IR neurons was greater in the SDN of ATD males as opposed to cholesterol males. The actual function of the SDN-POA neurons in controlling the expression of sexual behaviors is still not clear. Lesions restricted to the SDN had either no effect or a very small disruptive effect on masculine sexual behaviors in rats ${ }^{1,13}$ and ferrets, ${ }^{9}$ although in male gerbils lesions of the sexually dimorphic area strongly disrupted mating. ${ }^{36}$ The present data show that in both ATD and cholesterol males the matinginduced increments in Fos-IR occurred in portions of the mPOA which included, but were not restricted to, the SDN-POA. This is consistent with the results of lesion studies suggesting that there is no clear correlation between the function of SDN-POA neurons and the display of masculine sexual behavior in rats. ${ }^{1,13}$

\section{CONCLUSION}

The available evidence points to a sexually dimorphic processing in the central portion of the vomeronasal projection circuit of chemosensory cues derived from sexually active males as opposed to estrous females. The development of the sexually dimorphic neural response to male-derived odors results from the neonatal action of estrogen in the male rat's nervous system.

Acknowledgements-We thank Dr David Hancock for generously providing the DCH-1 Fos antiserum used in this study. M. J. Baum's contribution to this project was supported by U.S. Public Health Service grant HD21094 and by Research Scientist Award MH00392.

\section{REFERENCES}

1. Arendash G. W. and Gorski R. A. (1983) Effects of discrete lesions of the sexually dimorphic nucleus of the preoptic area or other medial preoptic regions on the sexual behavior of male rats. Brain Res. Bull. 10, 147-154.

2. Bakker J., van Ophemert J. and Slob A. K. (1993) Organization of partner preference and sexual behavior and its nocturnal rhythmicity in male rats. Behav. Neurosci. 107, 1049-1058.

3. Bakker J., Brand T., van Ophemert J. and Slob A. K. (1993) Hormonal regulation of adult partner preference behavior in neonatally ATD-treated male rats. Behav. Neurosci. 107, 480-487.

4. Bakker J., van Ophemert J., Timmerman M. A., de Jong F. H. and Slob A. K. (1995) Endogenous reproductive hormones and nocturnal rhythms in partner preference behavior and sexual behavior of ATD-males. Neuroendocrinology 62, 396-405.

5. Baum M. J. and Everitt B. J. (1992) Increased expression of c-Fos in the medial preoptic area after mating in male rats: role of afferent inputs from the medial amygdala and midbrain central tegmental field. Neuroscience 50, 627-646.

6. Baum M. J. and Wersinger S. R. (1993) Equivalent levels of mating-induced neural c-Fos immunoreactivity in castrated male rats given androgen, estrogen, or no steroid replacement. Biol. Reprod. 48, 1341-1347.

7. Brand T., Kroonen J., Mos J. and Slob A. K. (1991) Adult partner preference and sexual behavior of male rats affected Sby perinatal endocrine manipulations. Horm. Behav. 25, 323-341.

8. Bressler S. C. and Baum M. J. (1996) Sex comparison of neuronal Fos immunoreactivity in the rat vomeronasal projection circuit after chemosensory stimulation. Neuroscience 71, 1063-1072.

9. Cherry J. A. and Baum M. J. (1990) Effects of lesions of a sexually dimorphic nucleus in the preoptic/anterior hypothalamus area on the expression of androgen and estrogen-dependent sexual behavior in male ferrets. Brain Res. 522, 191-203. 
10. Davis P. G., Chaptal C. V. and McEwen B. S. (1979) Independence of the differentiation of masculine and feminine sexual behavior in rats. Horm. Behav. 12, 12-19.

11. De Jonge F. H. and van de Poll N. E. (1986) On the involvement of progesterone in sexually rewarded choice behavior of the female rat. Physiol. Behav. 37, 93-98.

12. De Jonge F. H., Eerland E. M. J. and van de Poll N. E. (1986) The influence of estrogen, testosterone, and progesterone on partner preference, receptivity and proceptivity. Physiol. Behav. 37, 885-891.

13. De Jonge F. H., Louwerse A. L., Ooms M. P., Evers P., Endert E. and van de Poll N. E. (1989) Lesions of the SDN-POA inhibit sexual behavior of male wistar rats. Brain Res. Bull. 23, 483-492.

14. Döhler K. D., Coquelin A., Davis F., Hines M., Shryne J. E. and Gorski R. A. (1982) Differentiation of the sexually dimorphic nucleus in the preoptic area of the rat brain is determined by the perinatal hormone environment. Neurosci. Lett. 33, 295-298.

15. Döhler K. D., Srivastava S. S., Shryne J. E., Jarzab B., Sipos A. and Gorski R. A. (1984) Differentiation of the sexually dimorphic nucleus in the preoptic area of the rat brain is inhibited by postnatal treatment with an estrogen antagonist. Neuroendocrinology 38, 297-301.

16. Everitt B. J. (1990) Sexual motivation: a neural and behavioural analysis of the mechanisms underlying appetitive and copulatory responses of male rats. Neurosci. Biobehav. Rev. 14, 217-232.

17. Gorski R. A., Gordon J. H., Shryne J. E. and Southam A. M. (1978) Evidence for a morphological sex difference within the medial preoptic area of the rat brain. Brain Res. 148, 333-346.

18. Guillamon A. and Segovia S. (1993) Sexual dimorphism in the accessory olfactory system. In The Development of Sex Differences and Similarities in Behavior (eds Haug M., Whaler R. E., Aron C. and Olsen K. L.), pp. 363-376. Kluwer Academic, Dordrecht, The Netherlands.

19. Hart B. L. and Leedy M. G. (1983) Female sexual responses in male cats facilitated by olfactory bulbectomy and medial preoptic/anterior hypothalamic lesions. Behav. Neurosci. 4, 608-614.

20. Hennessey A. C., Wallen K. and Edwards D. A. (1986) Preoptic lesions increase the display of lordosis by male rats. Brain Res. 370, 21-28.

21. Houtsmuller E. J., Brand T., de Jonge F. H., Joosten R. N. J. M. A., van de Poll N. E. and Slob A. K. (1994) SDN-POA volume, sexual behavior, and partner preference of male rats affected by perinatal treatment with ATD. Physiol. Behav. 56, 535-541.

22. Jacobson C. D., Csernus V. J., Shryne J. E. and Gorski R. A. (1981) The influence of gonadectomy, androgen exposure, or a gonadal graft in the neonatal rat on the volume of the sexually dimorphic nucleus of the preoptic area. J. Neurosci. 1, 1142-1147.

23. Kollack S. S. and Newman S. W. (1992) Mating behavior induces selective expression of Fos protein within the chemosensory pathways of the male Syrian hamster brain. Neurosci. Lett. 143, 223-228.

24. Kondo Y., Shinoda A., Yamanouchi K. and Arai Y. (1990) Role of septum and preoptic area in regulating masculine and feminine sexual behavior in male rats. Horm. Behav. 24, 421-434.

25. Mitchell J. B. and Gratton A. (1991) Opioid modulation and sensitization of dopamine release elicited by sexually relevant stimuli: a high speed chronoamperometric study in freely behaving rats. Brain Res. 551, $20-27$.

26. Olster D. H. (1993) Ibotenic acid-induced lesions of the medial preoptic area/anterior hypothalamus enhance the display of progesterone-facilitated lordosis in male rats. Brain Res. 626, 99-105.

27. Paredes R. G. and Baum M. J. (1995) Altered sexual partner preference in male ferrets given excitotoxic lesions of the preoptic area/anterior hypothalamus. J. Neurosci. 15, 6619-6630.

28. Robertson G. S., Pfaus J. G., Atkinson L. J., Matsumura H., Philips A. G. and Fibiger H. C. (1991) Sexual behavior increases c-Fos expression in the forebrain of the male rat. Brain Res. 564, 352-357.

29. Rodriquez-Sierra J. F. and Teresawa E. (1979) Lesions of the preoptic area facilitate lordosis behavior in male and female guinea pigs. Brain Res. Bull. 4, 513-517.

30. Sachs B. D. and Meisel R. (1988) The physiology of male sexual behavior. In The Physiology of Reproduction (eds Knobil E. and Neil J.), pp. 1393-1485. Raven, New York.

31. Segovia S. and Guillamon A. (1993) Sexual dimorphism in the vomeronasal pathway and sex differences in reproductive behaviors. Brain Res. Rev. 18, 51-74.

32. Swaab D. F., Slob A. K., Houtsmuller E. J., Brand T. and Zhou J. N. (1995) Increased number of vasopressin neurons in the suprachiasmatic nucleus ( $\mathrm{SCN}$ ) of 'bisexual' adult male rats following perinatal treatment with the aromatase blocker ATD. Devl Brain. Res. 85, 273-279.

33. Vreeburg J. T. M., van de Vaart P. D. M. and van der Schoot P. (1977) Prevention of central defeminization but not masculinization in male rats by inhibition neonatally of oestrogen biosynthesis. J. Endocr. 74, 375-382.

34. Wersinger S. R., Baum M. J. and Erskine M. S. (1993) Mating-induced Fos-like immunoreactivity in the rat forebrain: a sex comparison and a dimorphic effect of pelvic nerve transection. J. Neuroendocr. 5, 557-568.

35. West C. H. K., Clancy A. N. and Michael R. P. (1992) Enhanced responses of nucieus accumbens neurons in male rats to novel odors associated with sexually receptive females. Brain Res. 585, 49-55.

36. Yahr P. and Gregory J. E. (1993) The medial and lateral cell groups of the sexually dimorphic area of the gerbil hypothalamus are essential for male sexual behavior and act via separate pathways. Brain Res. 631, 287-296. 